\title{
Accurately Imaging, Tracking and Moving Single Atoms
}

Andrew Lupini ${ }^{1}$, Bethany Hudak ${ }^{2}$, Stephen Jesse ${ }^{1}$, Jiaming Song ${ }^{3}$, Ondrej Dyck ${ }^{1}$, Paul Snijders ${ }^{4}$ and Sergei Kalinin ${ }^{1}$

${ }^{1}$ Oak Ridge National Laboratory, Oak Ridge, Tennessee, United States, ${ }^{2}$ US Naval Research Laboratory, Washington, District of Columbia, United States, ${ }^{3}$ School of Physics, Northwest University, Xi' an, China, Xi'an, Xizang, China (People's Republic), ${ }^{4}$ Physical Review, Oak Ridge, Tennessee, United States

Single dopant atoms in a low-dimensional material, such as graphene, or near the surface of a bulk material such as silicon or diamond form a promising vehicle for many sorts of quantum device. However, positioning isolated dopant atoms in the area of interest, or even being able to tell precisely where they are remain as ongoing challenges. Aberration-corrected scanning transmission electron microscopy (STEM) provides a new view of these exciting materials.

One side effect of using an electron beam to image single atoms is that the beam transfers energy to the sample, which will often move the atoms of interest, although this process can allow new insight into familiar problems such as solid-state atomic diffusion [1]. In some cases, this energy transfer can be an advantage, opening new opportunities, such as using the precisely focused electron beam in a STEM to pattern materials at the atomic scale, with examples including controlling single atoms in two-dimensional materials [2,3] or sculpting oxides and crystalline materials with atomic plane resolution [4,5].

For these applications, it is desirable to be able to rapidly switch between imaging and manipulation modes. There are several strategies to vary the amount of energy imparted to the sample, such as scanning different patterns to vary the dose distribution [6] or by changing the beam energy. One complication of changing the accelerating voltage is that the main lenses in a high-resolution TEM are usually round magnetic lenses that require a lot of power. Scaling the lens currents for new accelerating voltages results in having to retune the aberration-corrector and leaves a long-term drift, which presents difficulties for continuous imaging or staying in the same area, as shown in Figure 1. Strategies such as maintaining a constant power in the lenses across different voltages offer a promising route to minimize these changes [7].

One difficulty with reducing the dose is that the signal to noise ratio of the resulting images is likely to be worse, making it harder to accurately locate and identify single atoms. Significantly, the precise mechanism of how atoms move under electron beam irradiation inside three-dimensional materials is yet to be uncovered [8]. Fast methods to analyze images and extract atomic coordinates (Figure 2) will allow the experimental data to provide input or verification of new theoretical models of the dynamics in these systems, with the goal of allowing better control over the positions of even single atoms. [9] 

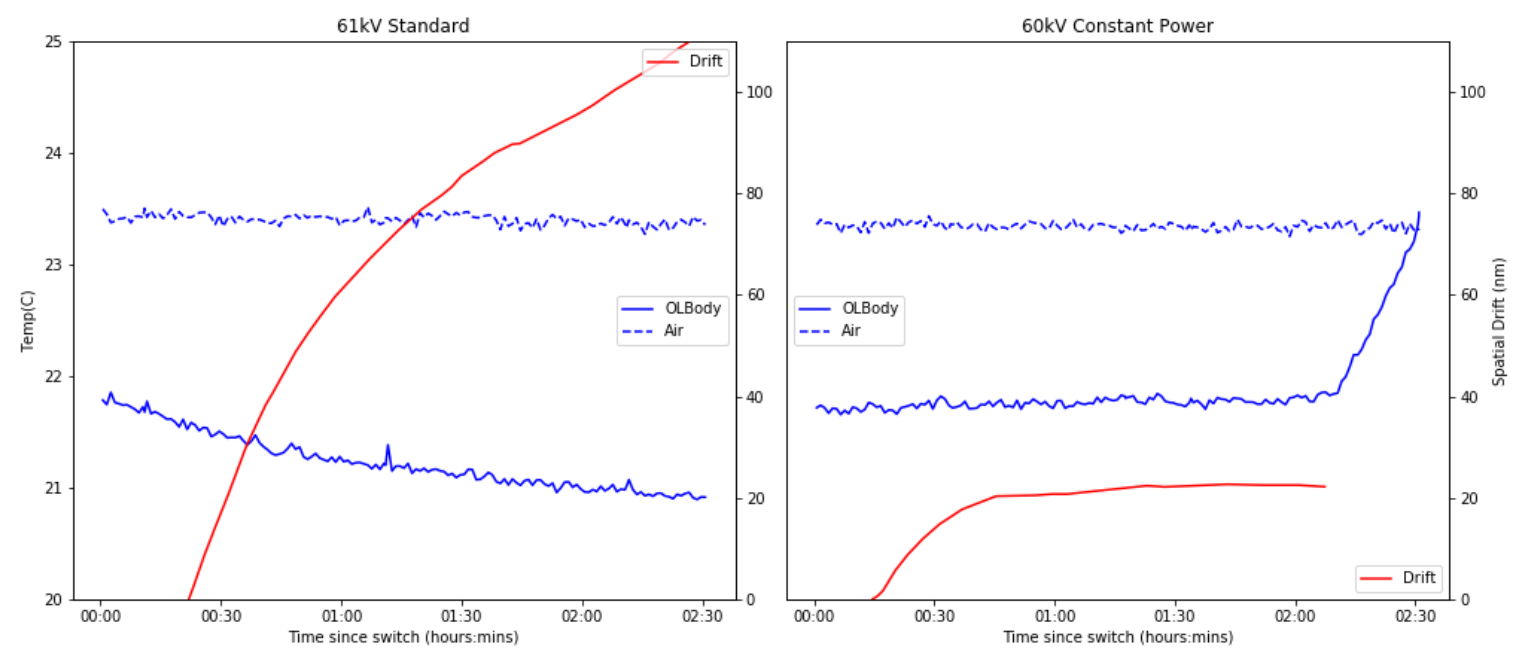

Figure 1. Comparison of spatial drift and temperature changes (measured at the objective lens body and inside the microscope enclosure) plotted over time following accelerating voltage changes from $100 \mathrm{kV}$. (Left) In the normal mode, temperature changes result in spatial drift. (Right) In the constant power mode, the nearly constant temperature results in significantly less drift. Reproduced from ref [7].
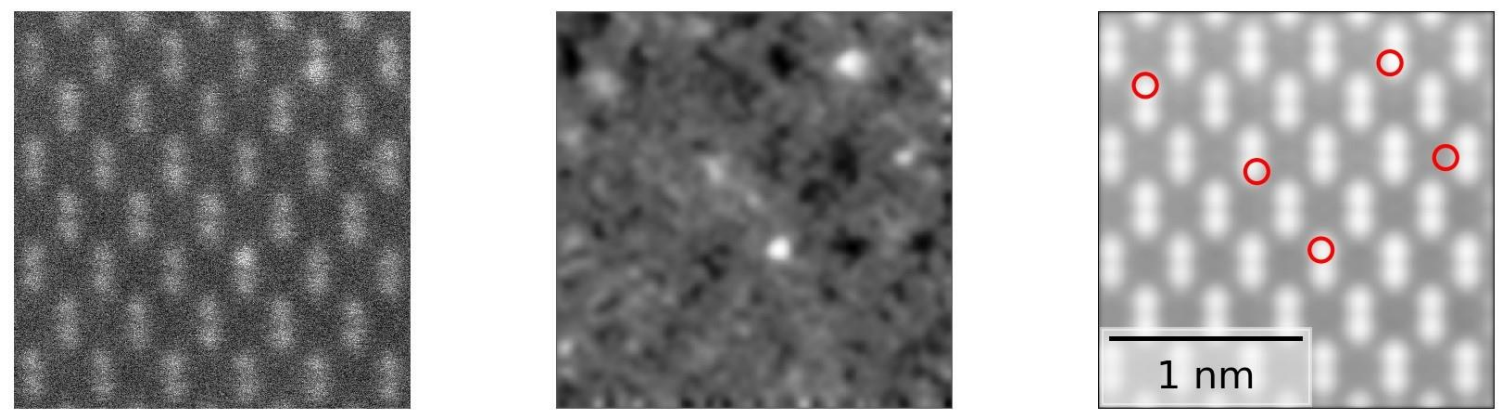

Figure 2. Attempt to automatically identify atomic positions in a frame extracted from a sequence of moving dopant atoms in silicon. (Left) Experimental image; (Center) intensity after drift correction and lattice background removal; (Right) most probable dopant positions marked on background.

\section{References}

[1] R Ishikawa et al, Phys Rev Lett 113 (15) (2014).

[2] T Susi et al., Ultramicroscopy 180 (2017), p. 163.

[3] O Dyck et al., Applied Physics Letters 111 (2017), p. 113104.

[4] S Jesse et al., Small 11 (2015), p. 5895.

[5] S Jesse et al., Nanotechnology 29 (2019), p. 255303.

[6] X. Sang et al, Scientific Reports 7 (2017) p. 43585

[7] O Dyck et al., Ultramicroscopy 211 (2020) p. 112949

[8] BM Hudak et al., ACS Nano 12 (2018), p. 5873.

[9] Work supported by the U.S. Department of Energy, Office of Science, Basic Energy Sciences, Division of Materials Science and Engineering, and by the Laboratory Directed Research and Development Program of Oak Ridge National Laboratory, managed by UT-Battelle, LLC for the U.S. Department of Energy, and was performed at the Oak Ridge National Laboratory's Center for Nanophase Materials Sciences (CNMS), a U.S. Department of Energy Office of Science User Facility. 